Bull. Mater. Sci., Vol. 21, No. 3, June 1998, pp. 251-255. (C) Indian Academy of Sciences.

\title{
An experimental setup for dynamic measurement of magnetoelectric effect
}

\author{
M MAHESH KUMAR, A SRINIVAS, S V SURYANARAYANA*, G S KUMAR and \\ T BHIMASANKARAM \\ Department of Physics, Osmania University, Hyderabad 500 007, India
}

MS received 13 October 1997; revised 17 February 1998

\begin{abstract}
An experimental setup is developed for the measurement of dynamic magnetoelectric effect (ME) in polycrystalline materials, using a time varying DC magnetic field on which an AC magnetic field is superimposed. The experimental data on $\mathrm{ME}$ on $\mathrm{Bi}_{5} \mathrm{FeTi}_{3} \mathrm{O}_{15}$ and a solid solution of $(90 \%) \mathrm{BiFeO}_{3}-(10 \%) \mathrm{BaTiO}_{3}$ are obtained using this setup. The linear and higher order ME coeficients can be evaluated from the output voltage. The temperature variation of $\mathrm{ME}$ data gives additional information corroborating with the data on magnetization.
\end{abstract}

Keywords. Dynamic magnetoelectric effect; ferroelectricity; bismuth layer structured ferroelectrics.

\section{Introduction}

The magnetoelectric effect (ME) in an insulator is the electrical polarization on applying $\mathrm{DC}$ or $\mathrm{AC}$ magnetic field $(\mathrm{ME})_{\mathrm{H}}$ or the magnetic polarization on the application of $\mathrm{DC}$ or $\mathrm{AC}$ electric field $(\mathrm{ME})_{\mathrm{E}}$. It occurs due to the interaction between the magnetic and electric dipoles. The condition for the realization of $\mathrm{ME}$ output is that there should exist electrical and magnetic ordering in the material at a given temperature and also that the electrical resistivity is reasonably high (Rado and Folen 1961, 1962; Freeman and Schmid 1975; Siratori et al 1992).

There are basically two kinds of materials which exhibit magnetoelectric output, namely single phase materials and composite materials. The single phase materials have an ordered lattice having two transitions corresponding to the ferroelectric to paraelectric, the other ferro/ferri/antiferro to paramagnetic state. While in the two-phase composites, one phase is piezomagnetic and the other piezoelectric. In the single phase material, $\mathrm{ME}$ output arises due to the interaction between the magnetic and ferroelectric sublattices (Suryanarayana 1994) while in the composite, ME output, a product property, is essentially due to the interaction between two participating phases (Van Suchtelen 1992; Suryanarayana et al 1992). Further, it is to be noted that in neither of the individual phases ME signal is observed. The synthesis of the materials is itself a challenging task since the impurity phases and interphases that may be formed in the composite and other defects, if any,

*Author for correspondence may also affect the signal. In the case of single phase materials, most of the known ME compounds have complicated crystal structures and show output at low temperatures. However, the best known ME materials like $\mathrm{Cr}_{2} \mathrm{O}_{3}$ and boracites were studied in the single crystal form (Astrov 1960; Folen et al 1961; Ascher et al 1966). In most other cases, the data on polycrystalline materials can still yield useful information to understand the structure-property correlations.

An important aspect of magnetoelectric phenomenon is the necessity to pole the samples both electrically and magnetically. A proper poling methodology would ensure dipole reorientations so that the sub-lattices (in the case of single phase material) or different constituent phases (in the case of composites) would effectively participate to yield reasonable polarization, to be detected as a voltage or charge output.

Both linear and higher order $\mathrm{ME}$ coefficients are possible, which have their origin in the expansion of density of stored free enthalpy $g(E, H, T)$ written in terms of a power series. The order of ME coefficient also depends on the symmetry to which the crystal belongs (Rivera 1994). The magnitude of ME output is very small but still well measurable by any of the following methods.

\section{Measurement techniques}

There are three methods of measuring ME output. (i) In the static method, ME output (charge or voltage) is measured as a function of increasing magnetic field using an electrometer having high input impedance. While poling, there is a possibility of charges to get accumulated 
at the grain boundaries which move towards the electrodes during the measurement. The discharge of the charges needs quite a lot of time, before actually the final output is stabilized (Hanumaiah et al 1994; Singh et al 1994; Suryanarayana 1994; James et al 1997). The accumulated charges in some materials may lead to erroneous conclusions as we inferred earlier in the case of $\mathrm{Bi}_{5} \mathrm{FeTi}_{3} \mathrm{O}_{15}$ (Singh et al 1994), (ii) for more precise data, quasi-static method is employed, which involves a time varying DC magnetic field and a high impedance electrometer. The output is measured as a function of time. This technique was used by Rivera (1994) in single crystal boracites and $\mathrm{Ye}$ et al (1994) in $\mathrm{Cr}_{3} \mathrm{~B}_{7} \mathrm{O}_{13} \mathrm{Cl}$. But this method cannot be employed for a polycrystalline material as the charge build up at the grain boundaries still affects the ME output and (iii) dynamic method.

\subsection{Dynamic method}

In the dynamic method, the output ME voltage is measured with a bias AC magnetic field while keeping the quasi-static measurement in act i.e. the measurement is carried out with a time varying DC magnetic field in the presence of an AC field. The bias AC magnetic field employed will not allow the charges to move towards the electrodes since a suitable signal with an appropriate frequency is used where the polarity of the signal changes with time. In what follows, the authors describe the details of dynamic method of measuring ME output that has been set up. The basic equations necessary to evaluate ME coefficients from the voltage are given below (Rivera 1994).

When a DC magnetic field is applied, ME output voltage $(V)$, in a polycrystalline material showing second-order effect can be given by the following equation,

$$
V \propto(\alpha H+\beta \cdot H \cdot H),
$$

where $\alpha$ and $\beta$ are the coefficients of linear and quadratic components of ME. Suppose that if an AC field $h_{0}$ is superimposed over the DC field $H_{0}$, then the effective field is

$$
H=H_{0}+h_{0} \sin (\omega t)
$$

A lock-in-amplifier tuned to the frequency $\omega(\omega=2 \pi f)$ would measure the output ME signal. Then we can write,

$$
V \propto\left(\alpha+\beta H_{0}\right) h_{0}=\alpha^{*}\left(H_{0}\right) h_{0} .
$$

When $H_{0}$ is zero, it is possible to find out the linear term $\alpha$ and hence the second-order term $\beta$ is evaluated in the presence of DC magnetic field. When $H_{0}$ is not zero, the above equation shows that $\alpha$ varies with $\beta$, giving rise to a pseudo-linear coefficient $\alpha^{*}\left(H_{0}\right)$.

\subsection{Experimental}

For the above measurement, a Helmholtz coil is mounted on the pole pieces of a DC electromagnet, whose magnetic field can be made time varying by interfacing it to a computer. The time varying DC magnetic field can be achieved by a programmable stepper motor attached to the potentiometer of the DC magnetic power supply. The block diagram is given in figure 1 .

A stepper motor with $0.7 \mathrm{~kg}-\mathrm{cm}$ torque and having

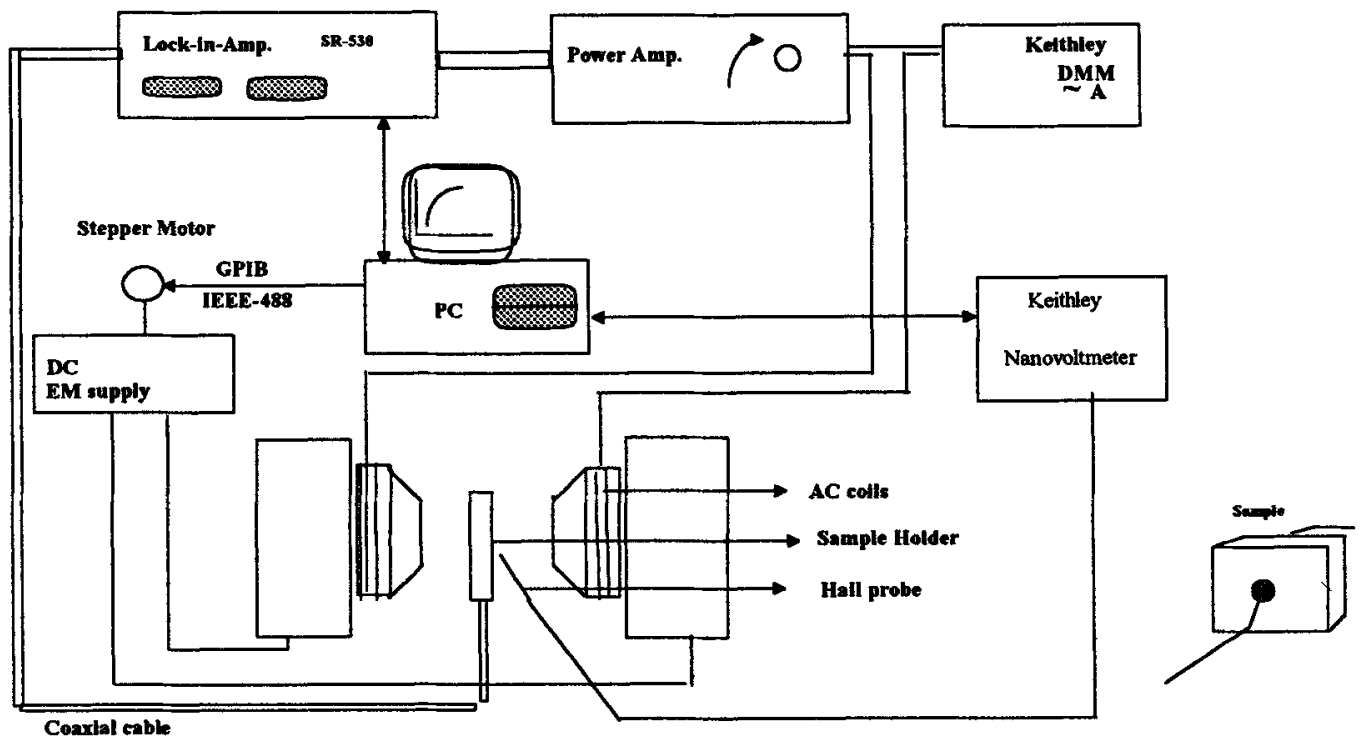

AC Magnetoelectric effect (Block Diagram)

Figure 1. Block diagram of dynamic magnetoelectric experimental setup. 
$1.2^{\circ}$ angle per rotation is used for the present purpose. A closed loop control system was achieved by measuring the output of the Hall probe in the Keithley 181 Nanovoltmeter and calibrating the Hall voltage with the field. Using the GPIB, it is possible to control the input power supply of the magnet using the combination of stepper motor and potentiometer. The AC Helmholtz coils each of 130 turns were mounted on the DC pole pieces. The ratio of the magnetic induction and the current through the coils is independent of the frequency. The coils are excited with a low-amplitude high-frequency $(1.006 \mathrm{kHz})$ signal, using the internal oscillator of the lock-in-amplifier (SR-530). Since the signal current was very small to drive the coils, a power amplifier was used to amplify the current. The AC magnetic field thus generated was calculated knowing the current carried by the coils using a Keithley 196 DMM. The whole setup was properly shielded to avoid stray pickups.

Keeping the DC magnetic field zero, with increasing AC field, the ME signal was recorded which gives the linear coefficient $(\alpha)$. Then by properly selecting a fixed value of $A C$ magnetic field, the DC field was swept using the stepper motor through the computer. The induced ME signal in the sample was measured with the lock-in-amplifier (SR530). With known values of $\alpha, h_{0}, H_{0}$ and $V$, the value of second-order coefficient $(\beta)$ can be calculated. Since, the total measuring time to complete the experiment is only few minutes, no time is available for the charges to accumulate in the sample, as in the case of pure $\mathrm{DC}(\mathrm{ME})_{\mathrm{H}}$ measurements. The signal output in voltage represents the true value. The same experimental setup, was used to take a temperature scan of the ME signal at fixed $\mathrm{AC}$ and $\mathrm{DC}$ bias fields by inserting the sample in a dewar in between the pole pieces. The data on $(\mathrm{ME})_{\mathrm{H}}$ at different temperatures would be useful to identify whether there are any magnetic anomalies in the material (Ikeda et al 1994).

Using the above experimental setup, AC (ME) measurements were carried out on the following materials, which have been studied earlier for their synthesis, characterization and $\mathrm{DC}(\mathrm{ME})_{\mathrm{H}}$ output in our laboratory (Singh et al 1994; Singh 1996; Mahesh Kumar et al 1998): (i) $\mathrm{Bi}_{5} \mathrm{FeTi}_{3} \mathrm{O}_{15}$ and (ii) (90\%) $\mathrm{BiFeO}_{3}-(10 \%)$ $\mathrm{BaTiO}_{3}$.

In addition to the data at room temperature (RT), measurements were also made at $77 \mathrm{~K}$. The temperature was measured using a Lakeshore silicon diode sensor DT-470. The samples were electroded with silver paint on both the sides and properly annealed. Two copper leads were attached on both the sides of the sample using epoxy resin. Prior to the measurement, the samples were poled electrically $\left(15 \mathrm{kV} / \mathrm{cm}\right.$ at $\left.110^{\circ} \mathrm{C}\right)$ and magnetically ( $3 \mathrm{kOe}$ at $\mathrm{RT}$ ).

The AC field $\left(h_{0}\right)$ employed was $18 \mathrm{Oe}$ at $1.006 \mathrm{kHz}$. A floating crystal was used to measure ME output and the differential inputs of the lock-in-amplifier were used to measure the output signal with coaxial cables. Along with the signal, the phase shift was also recorded. To minimize the noise due to cabling, a pre-amplifier SR-550 was included in the circuit.

As pointed out by Rivera (1994b), this experimental setup needs no calibration, contrary to $(\mathrm{ME})_{\mathrm{E}}$ method. Nevertheless, we have reexamined the data on $\mathrm{Bi}_{5} \mathrm{FeTi}_{3} \mathrm{O}_{15}$, which was investigated by Singh (1996).

\section{Results}

\section{$3.1 \mathrm{Bi}_{5} \mathrm{FeTi}_{3} \mathrm{O}_{15}$}

Figure 2 shows $\mathrm{ME}$ output of $\mathrm{Bi}_{5} \mathrm{FeTi}_{3} \mathrm{O}_{15}$. At room temperature, value of the output voltage decreased with increasing DC magnetic field (with AC field superimposed) up to $6 \mathrm{kOe}$. The shape of the curve is identical with that obtained by Singh (1996). It appears that the saturation fields are still higher. At $77 \mathrm{~K}$, while the nature of the output was similar to that at RT, with

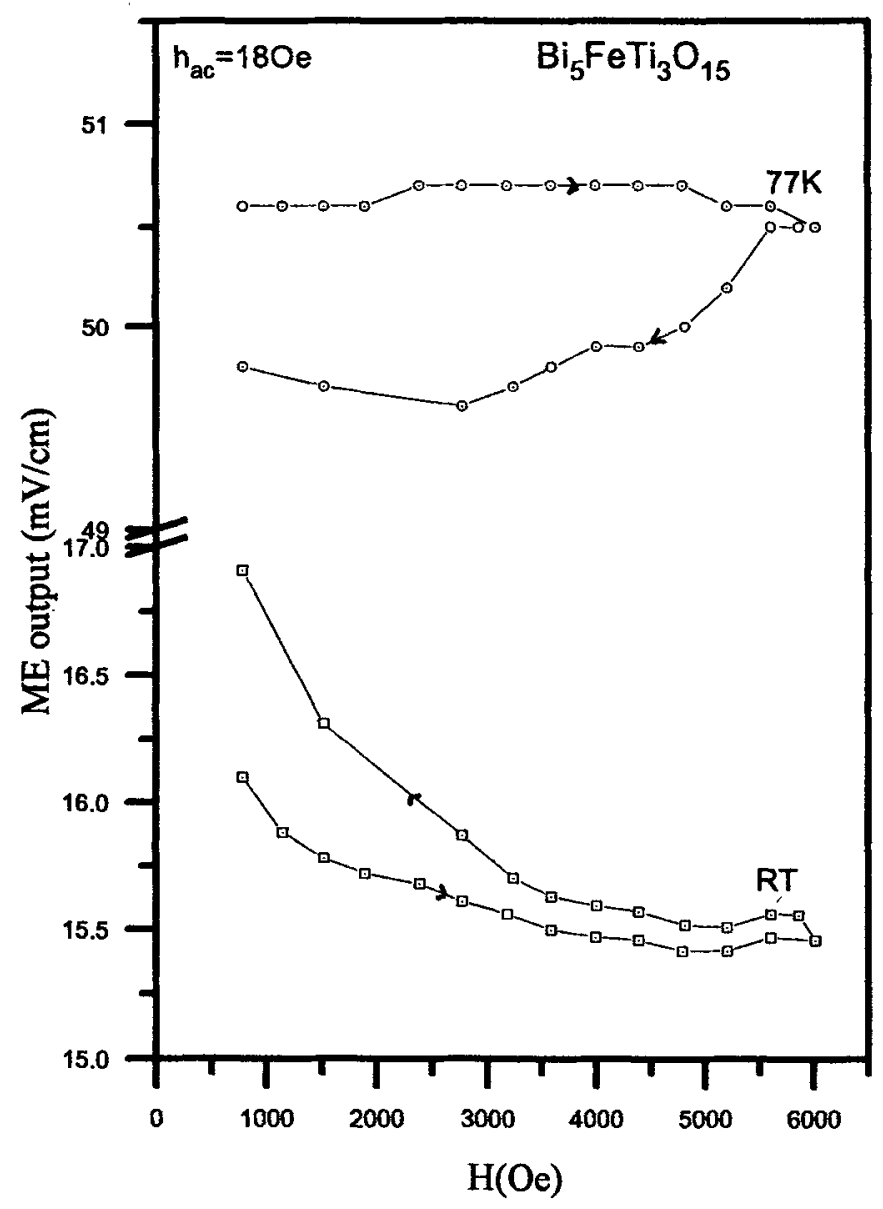

Figure 2. Variation of magnetoelectric output with DC bias magnetic field for $\mathrm{Bi}_{5} \mathrm{FeTi}_{3} \mathrm{O}_{15}$ at room temperature and $77 \mathrm{~K}$. 
increasing field, the difference between the low field and high field value is small. On reversal of the applied magnetic field, both the curves do not retrace the same path. The remanance at $R T$ is $16.9 \mathrm{mV} / \mathrm{cm}$, while at $77 \mathrm{~K}$ the value is $49.8 \mathrm{mV} / \mathrm{cm}$. The calculated values of $\alpha$ at RT and $77 \mathrm{~K}$ are $0.1 \mathrm{mV} / \mathrm{cm}-\mathrm{Oe}$ and $2.8 \mathrm{mV} / \mathrm{cm}$ -
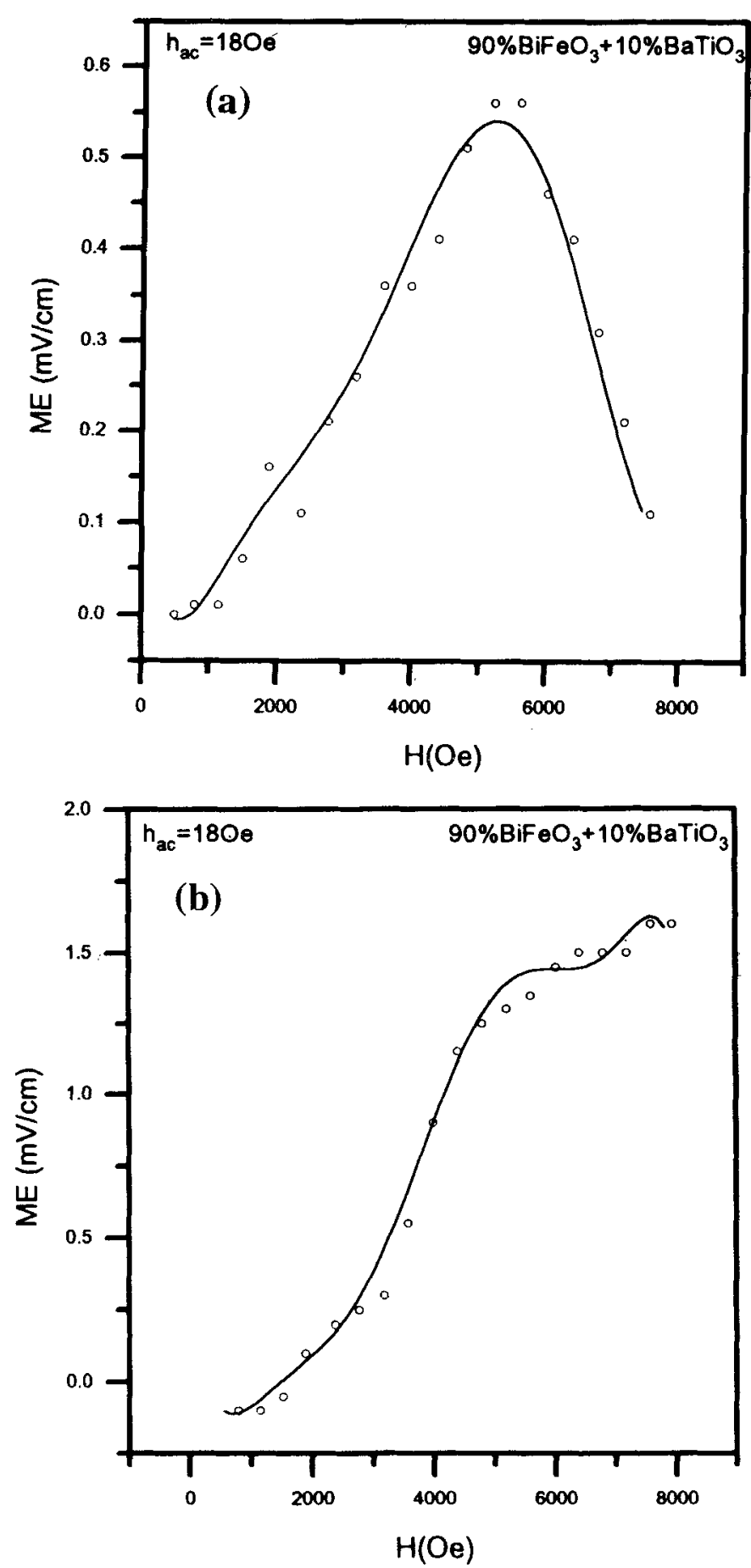

Figure 3. a. Variation of magnetoelectric output with $\mathrm{DC}$ bias magnetic field for $(90 \%) \mathrm{BiFeO}_{3}-(10 \%) \mathrm{BaTiO}_{3}$ at room temperature and $\mathbf{b}$. variation of magnetoelectric output with $\mathrm{DC}$ bias magnetic field for $(90 \%) \mathrm{BiFeO}_{3}-(10 \%) \mathrm{BaTiO}_{3}$ at $77 \mathrm{~K}$.
Oe, respectively. The value of linear coefficient increases with decreasing temperature. At RT the second order coefficient $(\beta)$ is $1.37 \times 10^{-8} \mathrm{mV} / \mathrm{cm}-\mathrm{Oe}^{2}$ and that at $77 \mathrm{~K}$ is $5.62 \times 10^{-9} \mathrm{mV} / \mathrm{cm}-\mathrm{Oe}^{2}$.

\section{$3.2(90 \%) \mathrm{BiFeO}_{3}-(10 \%) \mathrm{BaTiO}_{3}$}

Ismailzade et al (1981) have reported that $\mathrm{BiFeO}_{3}-\mathrm{BaTiO}_{3}$ forms a series of solid solutions with different structural modifications. It is rhombohedral between $100-67 \%$ of $\mathrm{BiFeO}_{3}, 67 \%$ to $7 \%$ cubic and from $7 \%$ tetragonal. They also reported magnetoelectric measurements at room temperature for the composition $95 \% \mathrm{BiFeO}_{3}-5 \% \mathrm{BaTiO}_{3}$. From the polarization vs magnetic field plot, they observed that the polarization increased with increasing field and at about $7 \mathrm{kOe}$, the output suddenly decreased. Recently, we have synthesized this system and reported its dielectric and electrical properties (Mahesh Kumar et al 1998).

Figure 3a shows $\mathrm{ME}$ output of $(90 \%) \mathrm{BiFeO}_{3}-(10 \%)$ $\mathrm{BaTiO}_{3}$ at RT. The value of the output increases with increasing DC field, reaches a maximum at $\sim 5.5 \mathrm{kOe}$ and later decreases. This field is lower than what Ismailzade et al (1981) have observed for $(95 \%) \mathrm{BiFeO}_{3}$ $(5 \%) \mathrm{BaTiO}_{3}$. Thus the mole\% of $\mathrm{BaTiO}_{3}$ in $\mathrm{BiFeO}_{3}$ has an effect on ME output and also on the magnetic field required to observe the peak in $\mathrm{ME}$ vs field measurement.

Figure $3 \mathrm{~b}$ shows $\mathrm{ME}$ output of the sample at $77 \mathrm{~K}$. ME output in this case peaks at $\sim 6 \mathrm{kOe}$. However, this peak is less pronounced than that observed at room

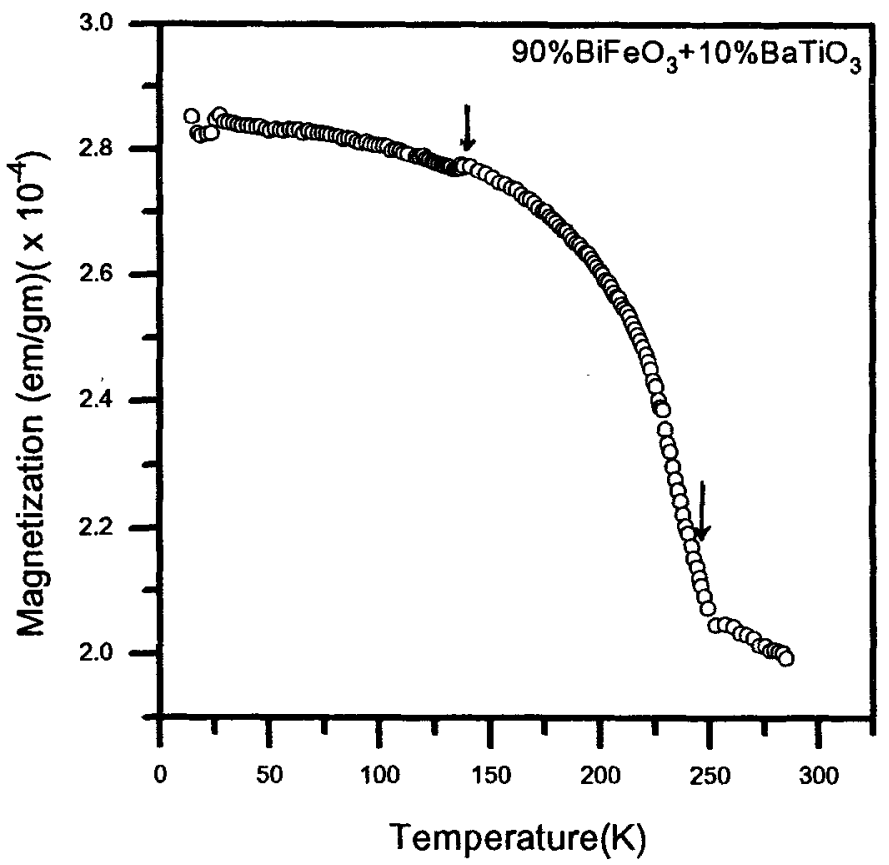

Figure 4. Variation of magnetization with temperature for $(90 \%) \mathrm{BiFeO}_{3}-(10 \%) \mathrm{BaTiO}_{3}$. 


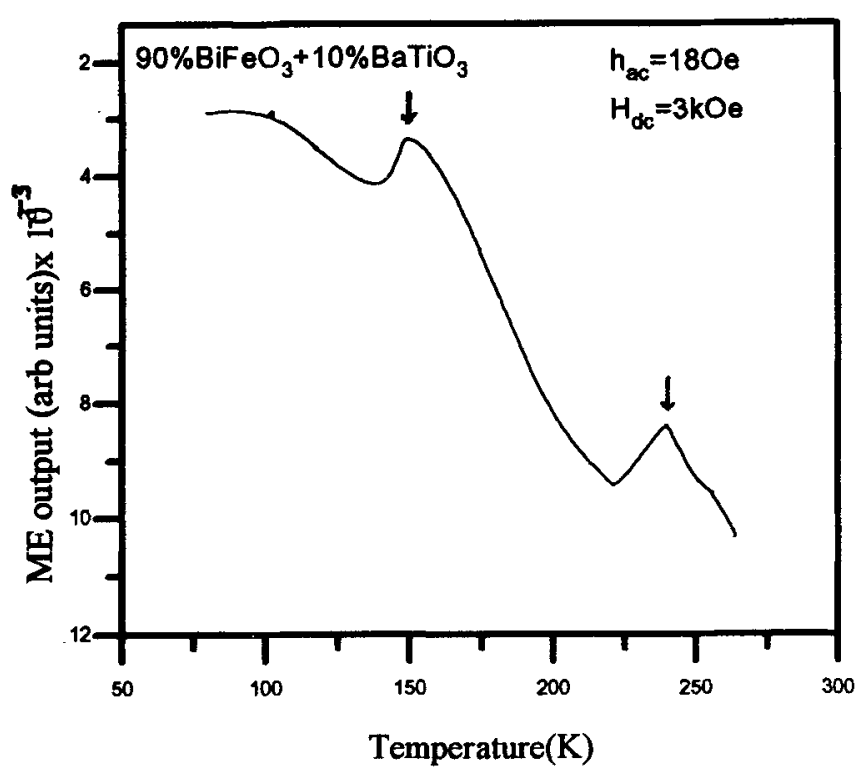

Figure 5. Variation of magnetoelectric output with temperature for $(90 \%) \mathrm{BiFeO}_{3}-(10 \%) \mathrm{BaTiO}_{3}$.

temperature. The maximum value of ME output obtained is $\sim 1.6 \mathrm{mV} / \mathrm{cm}$. The value of ME output has increased with decreasing temperature.

Figure 4 shows the magnetization vs temperature of the compound $(90 \%) \mathrm{BiFeO}_{3}-(10 \%) \mathrm{BaTiO}_{3}$. It is seen that the sample shows ferromagnetic nature, but as the temperature approaches $\mathrm{RT}$, the magnetization does not approach zero. It indicates that there may be yet another magnetic anomaly after the cross over from ferromagnetic state with a $T_{c}$ around $255 \mathrm{~K}$. Whereas there is a slope change around $140 \mathrm{~K}$, which may be attributed to the magnetic disorder in the system which appears at low temperatures.

Figure 5 shows the magnetoelectric output vs temperature for the sample measured at $h_{\mathrm{ac}}=18 \mathrm{Oe}$ and $H_{\mathrm{dc}}=$ $3 \mathrm{kOe}$ in the temperature range $77-300 \mathrm{~K}$. Anomalies were observed at $\sim 149 \mathrm{~K}$ and at $238 \mathrm{~K}$ corroborating with the slope changes in the magnetization data (figure 4), which indicates that the present experimental setup is useful not only to measure the ME output but also to corroborate small variations/fluctuations in the magnetization data.

\section{Conclusions}

A versatile dynamic technique to measure $\mathrm{ME}$ output has been setup. The technique can be used to perform a few additional experiments to yield the following data: (i) magnetoelectric output at room temperature and at different temperatures, (ii) from the data one can evaluate the linear and quadratic ME coefficients ( $\alpha$ and $\beta$ ), remanance and saturation fields, (iii) if the DC field is swept, both to positive and negative polarities, a butterfly loop can be traced between the applied field and output voltage, (iv) the phase angle of the output signal can be detected, which can be exploited for the possible device applications, (v) temperature scan of ME output can indicate possible magnetic transitions, and (vi) frequency scan at a particular temperature can also be made. A flat frequency response would be ideal for the material to be used as a transducer (Bracke and Van Vliet 1981).

\section{Acknowledgements}

The authors thank DRDO, Govt. of India for the financial assistance. MMK thanks CSIR for the award of a Senior Research Fellowship, Dr R S Singh (now at IISc, Bangalore) for many useful discussions in setting up the experiment and also thanks Prof. S N Kaul, University of Hyderabad, for providing facilities for magnetization measurements.

\section{References}

Ascher E, Rieder H, Schmid H and Stossel H $1966 \mathrm{~J}$. Appl. Phys. 371404

Astrov D N 1960 J. Exp. Theor. Phys. (USSR) 38984

Bracke L P M and Van Vliet R G 1981 Int. J. Electron. 51 255

Folen V T, Rado C T and Stalder E W 1961 Phys. Rev. Lett. 6607

Freeman A J and Schmid H (eds) 1975 Magnetoelectric interaction phenomena in crystals (London: Gordon and Breach)

Hanumaiah A, Bhimasankaram T, Suryanarayana S V and Kumar G S 1994 Bull. Mater. Sci. 17405

Ikeda N, Saito K, Kohn K, Kita H, Akimiku J and Siratori K 1994 Ferroelectrics 161111

Ismailzade I $\mathrm{H}$, Ismailov $\mathrm{R} \mathrm{M}$, Alekberov $\mathrm{A} I$ and Salaev F $\mathrm{M}$ 1981 Phys. Status Solidi (a) 68 K81

James A R, Kumar G S, Mahendra Kumar, Suryanarayana S V and Bhimasankaram T 1997 Mod. Phys. Lett. B11 633

Mahesh Kumar M, Srinivas A, Suryanarayana S V and Bhimasankaram T 1998 Phys. Status Solidi (a) 165317

Rado G T and Folen V J 1961 Phys. Rev. Lett. 7310

Rado G T and Folen V J 1962 J. Appl. Phys. 331126

Rivera J P 1994 Ferroelectrics 161 147, 165

Singh R S 1996 Structure-property correlations in some magnetically ordered Aurivillius phases, Ph D thesis, Osmania University, Hyderabad

Singh R S, Bhimasankaram T, Kumar G S and Suryanarayana S V 1994 Solid State Commun. 91567

Siratori K, Kohn K and Kita E 1992 Acta Phys. Polonica A81 4 Suryanarayana S V 1994 Bull. Mater. Sci. 171259

Suryanarayana S V, Sarah P, Singh R S, Bhimasankaram T and Mulay V N 1992 Proc. 5th Asia-Pacific physics conference, Kaula Lampur (Singapore: World Scientific) 2738

Van Suchtelen J 1992 Phillips. Res. Rep. 2728

Ye Z-G, Rivera J P, Schmid H, Heida M and Kohn K 1994 Ferroelectrics 16199 